\title{
Broad Band Equal-Length And Equal-Width Substrate Integrated Waveguide Four Channel Power Divider
}

\author{
Masoud Khoubroo Eslamloo', Pejman Mohammadi1 ${ }^{,}{ }^{*}$ \\ ${ }^{1}$ Department of Electrical Engineering, Urmia Branch, Islamic Azad University, Urmia, Iran \\ 2*p.mohammadi@iaurmia.ac.ir
}

\begin{abstract}
In this letter a novel broad band substrate integrated waveguide (SIW) power divider is proposed. It consist of four output channels made by SIW with equal length and equal width. Design equations and process are given with mathematical analysis. The propagation constant of the output signals have been adjusted by utilize only four via in the middle of the output arms. As a result a novel equal output power divider, is obtained accordingly. The experimental results of a prototype at $10 \mathrm{GHz}$ shows $3.1 \mathrm{GHz}$ bandwidth in both simulation and measurement results. Return loss and transmission coefficients have good agreement with simulation results in considered band.
\end{abstract}

Keywords: : Power divider, SIW, compact size 


\section{INTROCUCTION}

$\mathrm{T}$ he power divider is an essential component for realization of many microwave circuits. It is mainly used as feed network for array antennas and many other communication systems, such as wireless communication systems, radar systems, industrial and biomedical imaging systems. Wave guide power dividers have lower loss or dissipation in comparison to micro strip lines especially in higher frequencies of operation. [1]

Substrate integrated waveguide is a new structure which has become salient in recent decades [2,9] because of its wide impedance band width, compact size, low insertion loss and easily integration with other microwave component. $\mathrm{Y}$ and $\mathrm{T}$ shape power dividers [4,5], multi-way power dividers which are realized by combining the $\mathrm{Y}$ and $\mathrm{T}$ junctions have large size and high insertion loss. Radial cavity power dividers are presented as multi-way power dividers with compact dimension and lower insertion loss, however they have a non-planar feed structure [6,7] and narrow band width in resonant structures [6,7]. In this letter a novel four-port T-junction SIW power divider in which output arms have the same length and width has been presented as shown in Fig1. In other works structure have several input channels, however in this structure there is only one input as a feed port.

\section{ANALYSIS AND DESIGN}

SIW is a quasi-rectangular waveguide formed by two periodic via-hole connections between two metal layers.In the TE10 mode (dominant mode), SIW is equivalent to a conventional rectangular waveguide with negligible leakage losses. Therefore SIW and its equivalent dielectric field waveguide share the same TE10 mode cut-off frequency as follows:

$f_{\mathrm{c}}=\frac{1}{2 a \sqrt{\mu \varepsilon}}$

where $a$ is the width of the rectangular waveguide equivalent of SIW, $\mu$ and $\varepsilon$ are the permittivity and permeability of the substrate, respectively. The width of the rectangular waveguide equivalent

and fundamental properties of SIW are given approximately in [15] as follows:

$a=A-\frac{D^{2}}{0.95 P}$

where $\mathrm{a}$ is the width of the equivalent rectangular waveguide, $\mathrm{A}$ is the width of SIW, D is the diameter of metalized via holes and $\mathrm{P}$ is the pitch between adjacent via holeas as shown in Fig.2. The dimensions of the proposed power divider in Fig.1 are summarized in Table 1.

Four metalize inductive via have been added for dividing input signal equally between two arms and also matching the input port, for which the values of $(\mathrm{P})$ and (D) are $1.5 \mathrm{~mm}$ and $1 \mathrm{~mm}$ respectively. Also,Four additional vias (named as middle via) located just in front of each SIW arm are incorporated to improve return loss. Position of this four inductive vias are have important effect in return loss and transition coefficients of outputs. The output arms have same widths and same length. Therefore the propagation constant and the phase of the output signals will be the same. The designed power divider was etched on a piece of Rogers 4003 with $\varepsilon_{r}=3.55$, $\tan \delta=0.0027$ and thickness of substrate layer is $0.508 \mathrm{~mm}$.

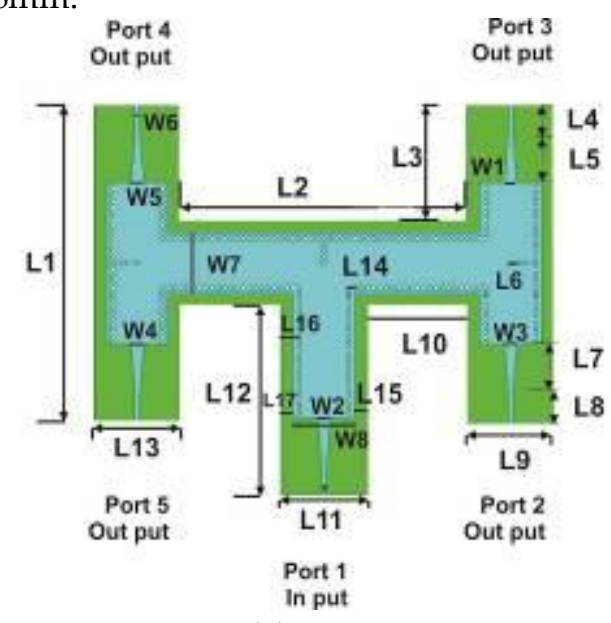

(a) 


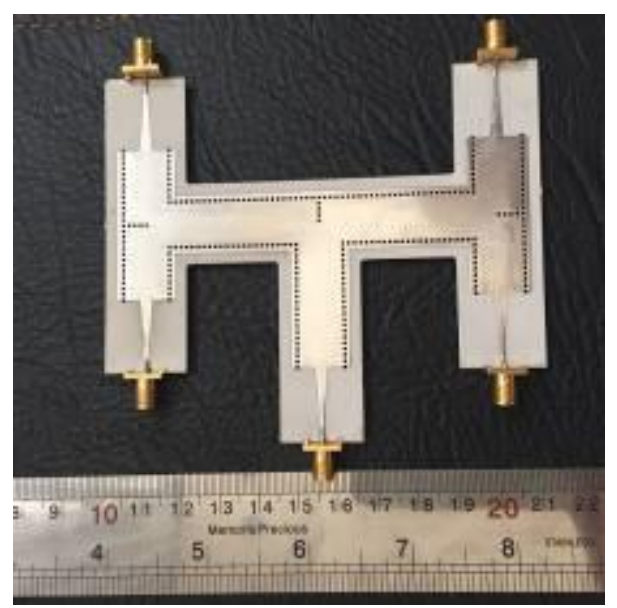

(b)

Fig.1. Geometry of (a) The proposed SIW power divider, (b) The fabricated structure

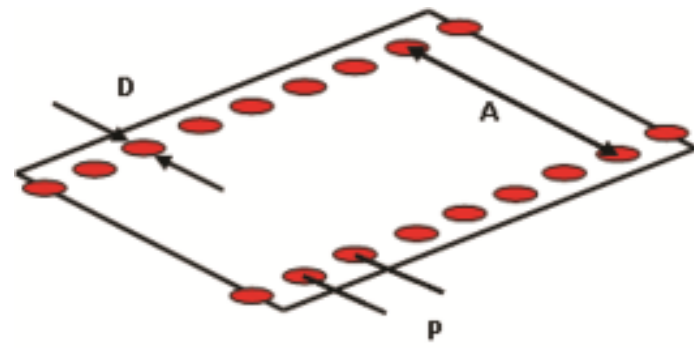

Fig.2. Substrate Integrated Waveguide Geometry

Table 1: Dimensions of the SIW power divider $(\mathrm{mm})$

\begin{tabular}{|cccc|}
\hline parametr & $\mathrm{mm}$ & parametr & $\mathrm{mm}$ \\
\hline $\mathrm{L} 1$ & 77 & $\mathrm{~L} 2$ & 69 \\
$\mathrm{~L} 3$ & 28 & $\mathrm{~L} 4-\mathrm{L} 8$ & 6.5 \\
$\mathrm{~L} 5-\mathrm{L} 7$ & 12.5 & $\mathrm{~L} 6$ & 1.5 \\
$\mathrm{~L}$ 9 & 21 & $\mathrm{~L} 10$ & 24 \\
$\mathrm{~L} 11-\mathrm{L} 13$ & 21 & $\mathrm{~L} 12$ & 46 \\
$\mathrm{~L} 14$ & 1.5 & $\mathrm{~L} 15-\mathrm{L} 17$ & 3 \\
$\mathrm{~L} 16$ & 4.5 & W1 - W2 & 3 \\
W3 & 3 & W4 & 3 \\
W5 & 3 & W8 & 15 \\
W6 & 1.1 & W7 & 15 \\
\hline
\end{tabular}

\section{MESUREMENT RESULTS}

CST MICROWAVE STUDIO software is used for full-wave simulation and optimization. Top view of the fabricated SIW power divider at the frequency of $10 \mathrm{GHz}$ are shown in Fig.1(b). The simulated and measured results of the S-parameters in the SIW power divider shown in Fig. $3(a, b)$ respectively, as can be seen bandwidth of 3.1 $\mathrm{GHz}$ - have been achieved for both simulation and measurement. The comparison between simulation and measurement results of the return loss are shown in Fig.3(c). The differences between the results of measurement and simulation are due to the effects of the SMA connectors soldered into structure and the fabrication process. Comparison between the measurements results of this work with some references are given in table 2.

According to simulation and measurement results for transmission coefficients in Fig.4 the output power levels are the same. There are a little difference between simulation and measurement results which may be outcomes from connectors and fabrication process. The output signals are in phase as expected and which are shown in Fig.5

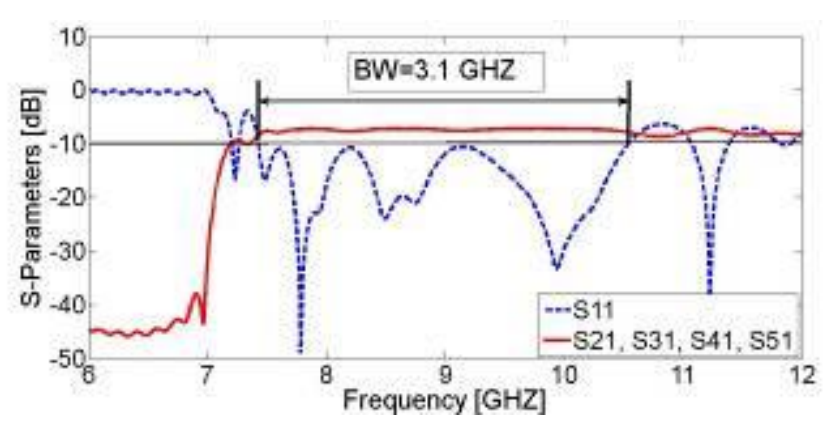

(a)

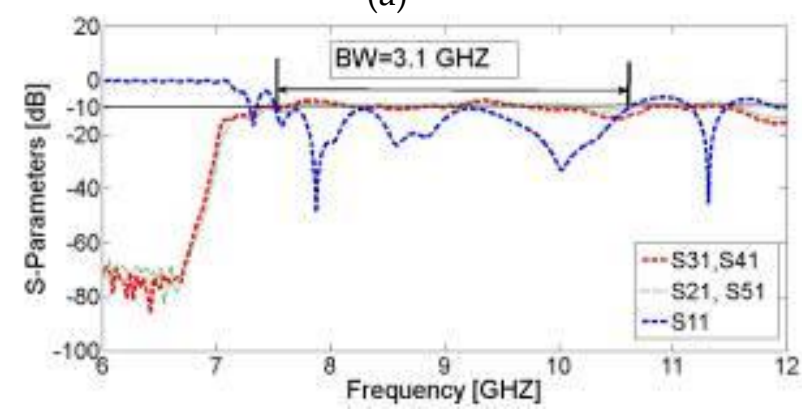

(b)

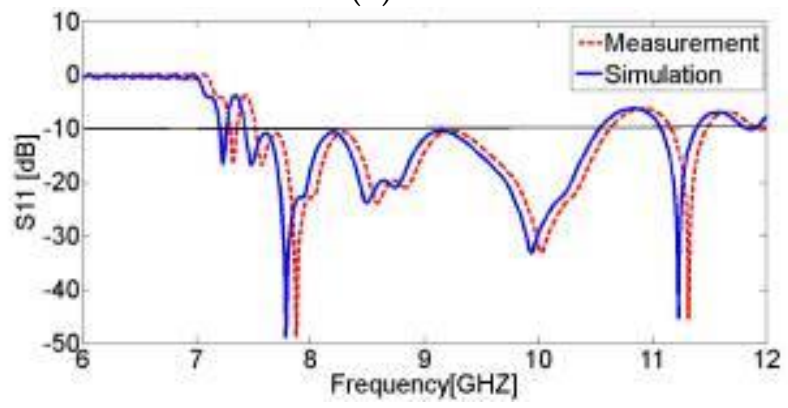

(c)

Fig 3. The S-parameters of SIW power divider (a) simulation (b) measurement (c) $S_{11}$ simulation and measurement 
Table 2: Compression between this work and some other works

\begin{tabular}{|c|c|c|c|c|}
\hline Ref.r & Bandwidth Frequency [GHz] & Fractional bandwidth & Transition [dB] & Size [mm2] \\
\hline$[10]$ & $7.3-13$ & 57 & $-7.6 \tan =0.001$ & $65^{\star} 65$ \\
{$[11]$} & $4.5-6.8$ & 40 & $-6.7 \tan =0.0009$ & $80^{\star} 140$ \\
{$[12]$} & $8-11$ & 27 & $\ldots \ldots \ldots \ldots$. & $107^{\star} 107$ \\
{$[14]$} & $12.3-15.5$ & 23 & $-7.1 \tan =0.0009$ & $45^{\star} 110$ \\
{$[14]$} & $12.3-15.5$ & 22 & $-6.6 \tan =0.0009$ & $50^{\star} 50$ \\
[13] & $4-11$ & 93 & $\ldots \ldots \ldots$ & $165^{\star} 165$ \\
[This work] & $7.6-10.7$ & 32 & $-7.2 \tan =0.0027$ & $77^{\star} 111$ \\
\hline
\end{tabular}

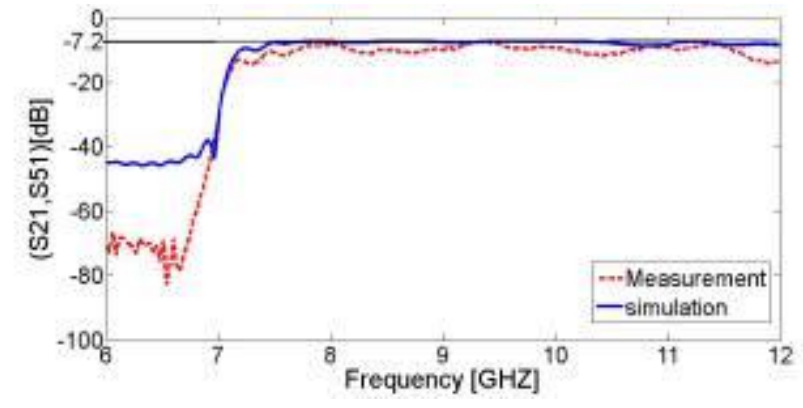

(a)

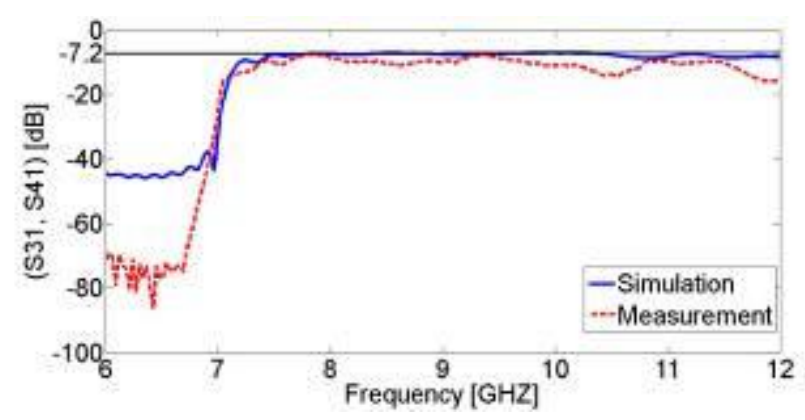

(b)

Fig.4. Transmission coefficients of Output Channels(a) compression between measurement and simulation results in second and fifth channels (b) compression between measurement and simulation results in third and fourth channels

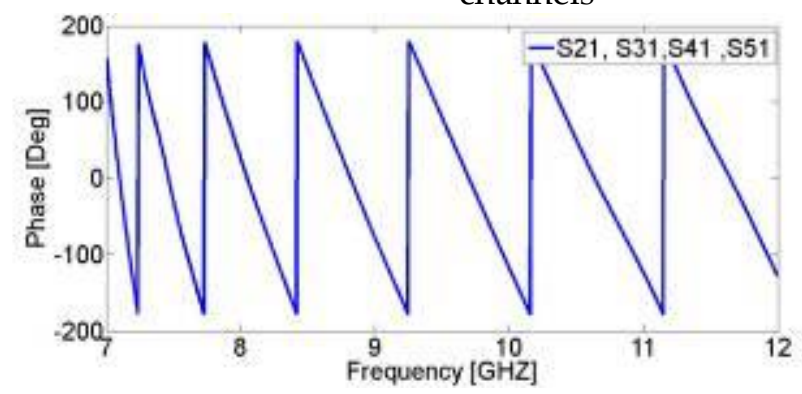

(a)

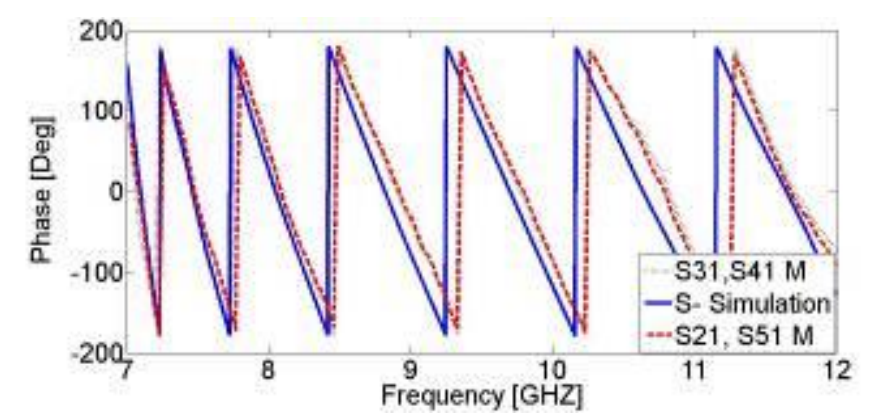

(b)

Fig.5. Phases of Output Channels(a) simulation (b) compression between measurement and simulation results

\section{CONCLUSION}

A novel power divider based on SIW technology with the equal length and equal width for output arms is proposed. The proposed SIW power divider has one input and four equal length and width outputs, therefore it has compact size in comparison with available SIW power dividers. Detailed of the design procedure is completely explained. The correctness of the analytical approach is validated by a prototype SIW power divider. Therefore such a SIW power divider is a good candidate for development of a compact size and integrated microwave systems.

\section{REFRENCES}

[1] Wu, K., Deslandes, D., and Cassivi, Y.: 'The substrate integrated circuits - a new concept for high-frequency electronics an doptoelectronics'. 6th Int. Conf. on Telecommunications in Modem Satellite, Cable and Broadcasting Service, 2003, 
(TELSIKS 2003),Nis, Serbia and Montenegro, October 2003, Vol. 1, pp. 3-5

[2] Germain, S., Deslandes, D., and Wu, K.: 'Development of substrate integrated waveguide power dividers'. IEEE CCECE Canadian Conf. on Electrical and Computer Engineering, Montreal, Canada, May 2003, Vol. 3, pp. 1921-1924

[3] Songnan, Y., and Fathy, A.E.: 'Synthesis of an arbitrary power split ratio divider using substrate integrated waveguides'. IEEE MTTS Int. Microwave Symp. Dig. (IEEE MTT-S), Honolulu, HI, USA, June 2007, pp. 427-430

[4]S. Germain, D. Deslandes and K. Wu, "Development of substrate integrated waveguide power dividers," CCECE 2003, pp. 1921-1924.

[5]Z. C. Hao, W. Hong, H. Li, H. Zhang and K. $\mathrm{Wu}$, "Multiway broadband

substrate integrated waveguide (SIW) power divider," 2005

International Symposium on Antenna and Propagation, pp. 639-642.

[6]K. Song, Y. Fan and X. Zhou, "X-band broadband substrate integrated rectangular waveguide power divider," Electronics Lett., vol. 44, pp. 211-213, Jan. 2008.

[7]K. Song and Y. Fan, "Broadband travellingwave power divider based on substrate integrated rectangular waveguide," Electronics Lett., vol. 45, pp. 631-632, Jun. 2009.

[8]K. Song, Y. Fan and Y. Zhang "Radial cavity power divider based on substrate integrated waveguide technology," Electronics Lett., vol. 42,pp. 1100-1101, Sep. 2006.

[9]K. Song, Y. Fan and Y. Zhang, "Eight-way substrate integrated waveguide power divider with low insertion loss," IEEE Trans. Microw.Theory Tech., vol. 56, pp. 1473-1477, Jun. 2008.

[10] X. Zou, C. M. Tong and D. W. Yu "Yjunction power divider based on substrate integrated waveguide," Electronics Lett., vol. 47, pp. 13751376, Dec. 2011.

[11] S.Karimabadi, A. Attari, "Broadband Substrate Integrated Waveguide Four-way
Power Divider," 6'th International Symposium on Telecommunications,IEEE 978-1-4673-2073-3/12, (IST'2012) 2012

[12] Sohum Datta, Soumava Mukherjee, AnimeshBiswas, "Design of Broadband Power Divider based on Substrate-Integrated Waveguide Technology," IEEE 978-1-47993267-2/13, 2013

[13] Lei Zhang, Xiaowei Zhu, Peng Chen, Ling Tian and Jianfeng Zhai, "Broadband FourWay Power Divider for Active Antenna Array Application ," State Key Lab of Millimeter Wave, Southeast University, Nanjing, 210096, P. R. China

[14] Bing Liu, Wei Hong, Lin Tian, Hong-Bing $\mathrm{Zhu}$, Wei Jiang and $\mathrm{Ke} \mathrm{Wu}$, "Half Mode Substrate Integrated Waveguide (HMSIW) Multi-way Power Divider," Proceedings of Asia-Pacific Microwave Conference 2006

[15] X.-P. Chen and K. Wu, "Substrate Integrated Waveguide Filter: Basic Design Rules and Fundamental Structure Features," Microwave Magazine,IEEE, vol. 15, pp. 108-116, July 2014 